\section{COVID-19 and taking care and protection of patients with intellectual disabilities, need special care and equity}

\author{
Faheem Anwar ${ }^{1}$, Muhammad Tayyab ${ }^{2,3 *}$, Jawad Khan ${ }^{1}$ and \\ Ihteshamul Haq ${ }^{1}$
}

${ }^{1}$ Department of Genetics, Hazara University, Mansehra, Pakistan ${ }^{2}$ Centre of Organismal Study (COS), University of Heidelberg, Germany

${ }^{3}$ Institute of Biotechnology and Genetic Engineering (IBGE), Agriculture University, Peshawar, Pakistan

Since December 2019, entire world is facing problem of corona-virus pandemics and its impact on the people and their social life has been phenomenal. Each part of the world is 'almost' hit by COVID-19 infection. Most of the COVID-19 victims were aged people followed by consequence of high death ratios as shown in data [1]. Not only aged people but people with some secondary diseases or disorder were of major concern. A special case comes across which are patients with intellectual disabilities (ID) are the most vulnerable group. They also have extra multiple disorders including respiratory diseases, diabetes, obesity, These individuals face more complications and stand at high risk of because, such people are usually mentally lethargic and have almost no literacy in to follow proper health care and access health facilities [2]. A new review on the psychological effects of quarantine based on an indication from the past pandemics stated that living with symptoms of corona is often linked with social behavior such as shock, anger, and confusion, perhaps if exists for long time [3]. Based on the above data it can be inferred that family members of patients with intellectual disabilities (ID) many times feels a huge pressure from the society ignore the critics from the surrounding people and thus limit their access to the available information find it difficult to grasp it. Based on these facts it can be inferred that sometimes they are unable to follow public health advice. Ultimately, the disease can spread easily to people in the same environment. Patients of intellectual disabilities (ID) always live in a shared community with family members as well as with caregivers, because of their mental retardness and being physically disable make them totally dependent on others. That's why the risk of this pandemic's spread becomes more marked both to patients with intellectual disabilities and their caregivers because intimacy to patients. The family members can't let patients with intellectual disabilities alone although

\section{More Information}

*Address for Correspondence:

Muhammad Tayyab, Centre of organismal study (COS), University of Heidelberg, Germany, Email: faheem_genetics@hu.edu.pk

Submitted: 05 October 2020

Approved: 12 October 2020

Published: 13 October 2020

How to cite this article: Anwar F, Tayyab M, Khan J, Haq I. COVID-19 and taking care and protection of patients with intellectual disabilities, need special care and equity. Int J Clin Virol. 2020; 4: 116-117

DOI: 10.29328/journal.ijcv.1001026

Copyright: ( 2020 Anwar F, et al. This is an open access article distributed under the Creative Commons Attribution License, which permits unrestricted use, distribution, and reproduction in any medium, provided the original work is properly cited.

\section{D) Check for updates}

(.) Open Access

additionally if they are reported confirmed positive for COVID-19. Because patients will be dependent for food and medicines on family members. So social distancing will not be possible in such special cases. Some secondary symptoms were also seen in the time of such pandemics in patients with intellectual disabilities. If they are ignored or not given proper attention and ultimately develops general sensitivity. This COVID-19 created a serious social issue because people maintain social distancing and health challenge that affect mental health if existed for long term may be extremely stressful. Being members of a very close family may face more problems and may worsen the conditions of the patients. Being ignorance and losing care from loved ones and caregiver for long term i.e. Family members who have been seriously ill with the coronavirus and they may avoid encountering them can potentially increase anxiety and frustrations. It will have a direct impact on their behavior as well. Special help will be needed from social workers and health carers to figure out a way of assisting families of patient's with intellectual disabilities with high risk. In this way they can provide a flexible way to support and assist them and can prevent severe damage. Amongst other symptoms developed of patients with intellectual disabilities are Fatigue, psychiatric disorders, frustration, fear, and anxiety about their loved ones. The caregiver should also try to facilitate the patients to their maximum capacity within the limits of government policies as well. There is a need to target individual, professional, health, and multiple levels of social welfare and local and national 
care system within law. The health social workers are needed to help increase public health and literacy with people with disabilities at this critical time. They also need to recognize the symptoms to help someone with an illness, discomfort, or developmental disorders. Patients with intellectual disabilities during pandemics need special accommodation facilities who are at great risk for outbreaks. They are usually neglected by both medicals and caregiver to transfer special and transparent knowledge how to take care of such patients during pandemics is the need of the day. Many workers, organizations along with medical practitioners who handle corona-virus patients in emergency during critical period, are specially advised to take special care to such patients along with providing them psychiatric facilities.

No specific treatment not only for normal people affected with coronavirus are there yet, Hence patients of intellectual disabilities positive for corona virus will need further strategies. The medicine they use for their intellectual disabilities (ID) should be properly checked not to have an adverse effect if use along with their normal routine medicines. Looking to all these cases rising with patients of intellectual disabilities needs a serious care system although seems difficult option, such as use of ventilator when lacking resources. Conclusion is that these patients at risk must be supported by people from different fields having different expertise in medical care system. We also need to gather a conclusive information throughout the pandemics from different patients of Intellectual disabilities, in terms of severances of diseases, medicines used, symptoms and if they have defeated coronavirus. These people will enhance the transparency of the information. On the other hand, progress and development will move forward to implement appropriate involvements must be adopted for future health events that cause a lot of harm to such vulnerable people.

\section{References}

1. Weiss $P$, Murdoch DR. Clinical course and mortality risk of severe COVID-19. The Lancet. 2020; 395: 1014-1015.

PubMed: https://pubmed.ncbi.nIm.nih.gov/32197108/

2. Glover G, Williams R, Heslop P, Oyinlola J, Grey J. Mortality in people with intellectual disabilities in England. J Intellect Disabil Res. 2017;61:62-74. PubMed: https://pubmed.ncbi.nlm.nih.gov/27489075

3. Brooks SK, Webster RK, Smith LE, Woodland L, Wessely S, et al. The psychological impact of quarantine and how to reduce it: rapid review of the evidence. The Lancet. 2020. 